\title{
Qualitative Alterations on Corneal Endothelial Cell Morphometry and Hexagonality After Cataract Surgery
}

\author{
Francesco Saverio Sorrentino \\ Department of Surgical Sciences, Unit of \\ Ophthalmology, Ospedale Maggiore, \\ Bologna, Italy
}

\begin{abstract}
Aim: To deeply analyze quantitative and qualitative changes of corneal endothelium after longitudinal phacoemulsification.

Methods: In this prospective interventional case series study, 50 eyes with age-related cataract have been evaluated preoperatively, intraoperatively and postoperatively. The measured parameters were surgically induced endothelial cell loss (ECL), average endothelial cell area (AVG) and hexagonality (HEX).

Results: The relationship among the measured parameters and the energy dissipated during the two phases of phacoemulsification, sculpting and quadrant removal, has been analyzed with regard to the 5-score harm scale, a new method suggested to categorize cataracts. Two phases of phacoemulsification are linearly related $\left(\mathrm{r}=1.5, \mathrm{P}<0.001, \mathrm{r}^{2}=79 \%\right)$. Plus, a quadratic model described the correlation between the percentages of ECL and AVG $(\mathrm{P}<$ 0.0001), while there was no specific model for the correlation between ECL and HEX.

Conclusion: The 5-score harm scale allows to predict the mean changes in percentages of ECL, AVG and HEX of endothelial cells after longitudinal phacoemulsification. Also, this study confirms that the main damage on corneal endothelium is due to the energy delivered in the second phase of phacoemulsification.
\end{abstract}

Keywords: phacoemulsification, cataract, endothelial cell loss, average cell area, hexagonality

\section{Introduction}

The safety of cataract surgery has markedly improved due to novel techniques, new machines and helpful viscoelastic devices. ${ }^{1}$ The integrity of the capsular bag and the care of corneal endothelium are the main concerns of surgeons. ${ }^{2}$ Some surgical maneuvers and several intraoperative mechanical factors might provoke alterations within the anterior chamber, potentially followed by harm on corneal endothelium. Using the conventional longitudinal phacoemulsification (phaco) setting mode, the ultrasound power is generated by longitudinal excursions of the phaco tip, provoking a sort of jackhammer effect. ${ }^{3}$ In previous studies, non-complicated cataract surgery has been observed to induce endothelial cell loss ranging from $12 \%$ to $20 \%{ }^{4-6}$

The corneal endothelium is a monolayer of hexagonal cells that lines the posterior corneal surface. ${ }^{7}$ During life, the central endothelial cell density gradually decreases at an average rate of about $0.6 \%$ per year going approximately from 3400 cells $/ \mathrm{mm}^{2}$ at age of 15 to 2300 cells $/ \mathrm{mm}^{2}$ at age of 85 years. ${ }^{8,9}$ Corneal endothelial cells (ECs)
Correspondence: Francesco Saverio Sorrentino

Email dr.fsorrentino@gmail.com 
are stuck in the G1 phase of the cell cycle, so they cannot proliferate during life. In case of endothelial injuries, for instance after cataract surgery, the survived ECs spread out, changing size and shape over the time. ${ }^{10}$ The healthy endothelium acts as a barrier having a "pump-leak" function, which enables to maintain corneal transparency and well-balanced stromal hydration. ${ }^{7,10}$ Non-contact specular and confocal microscopy are extremely useful machines to clinically investigate and provide for a morphological evaluation of the corneal endothelium. ${ }^{11,12}$

The 5-score harm scale, first discussed by Sorrentino et al in 2016 and 2017, is a new method to categorize cataracts according to preoperative and intraoperative variables (grade of hardness and phacoemulsification times). The challenging aim is to try to establish a connection between the type of cataract and the predictable harm on corneal endothelium after longitudinal phacoemulsification. According to the 5-score harm scale, on which further investigation is currently in progress, this study will analyze changes of corneal endothelium in morphology and hexagonality, assessing the effect of the total energy delivered into the anterior chamber after longitudinal phaco. Plus, we would like to show that the most energy is delivered throughout the quadrant removal phase. Statistical analysis has been used to describe the relationships between phases of phaco and quantitative/ qualitative changes of ECs.

\section{Subjects and Methods}

This prospective interventional study included fifty subjects (50 eyes) and was carried out in the Clinic of Ophthalmology at Ferrara University Hospital. The tenets of the Declaration of Helsinki were followed. This study was approved by Local Ethics Committee of Ferrara University and patients signed informed consent for the use of their data for research.

Patients are not consecutively selected over a period of three months in the outpatient office for cataract surgery. Each grade of age-related cataract was an inclusive criterion to have surgery that has been performed with the conventional longitudinal phaco setting mode. Patients were examined four weeks before cataract surgery and followed for six weeks after surgery. Patients with any ocular comorbidities (ie, pseudoexfoliation) or corneal abnormalities (ie, Fuchs dystrophy) that could affect corneal topography measurements were ruled out.

Likewise, in other studies conducted in 2016 and 2018 by Sorrentino et al, the non-contact specular microscopy
EM-3000 (Tomey GmbH, Erlangen, D) was used to register the following parameters preoperatively and postoperatively: endothelial cell density (ECD), average endothelial cell area (AVG), and percentage of hexagonal cells (HEX). ${ }^{6}$ To analyze our data, we calculated the differences in percentage between pre-op and post-op for each variable, namely ECL\%, AVG\% and HEX\% [(postop - preop/preop) $\times 100$ ], keeping into consideration the absolute value. According to the Lens Opacities Classification System II (LOCS II), the nucleus density grade was preoperatively estimated at slit lamp. ${ }^{13}$ The experienced surgeon set the phaco machine (Optikon R-Evolution - Optikon 2000 spa, Rome, IT) on the longitudinal energy mode and performed cataract surgery applying the "divide and conquer nucleofractis" technique. ${ }^{14}$ There were no significant complications nor intraoperative nor postoperative.

Intraoperative measurements, that is phaco times, followed the same working protocol already published by Sorrentino et al in their pilot study. ${ }^{6}$ Two aspects to note: first, the surgeon could have linear control of the phaco power by foot pedal; second, the maximum ultrasound power for each phase of phaco was preset. ${ }^{15,16}$

Data were analyzed with the MINITAB 17 software (MINITAB Inc., Pennsylvania State College, USA). A descriptive analysis was performed and the normality test of the data was carried out. The one-way ANOVA test was used to compare the quantitative/qualitative effects on ECs among the different scores of the harm scale. The value of $\mathrm{P}<0.05$ was considered for statistical significance.

There are no restrictions on the availability of data. Readers can freely obtain materials and information about this study just by asking the author.

\section{Results}

The first graph in Figure 1 (left) shows the increasing trend of ECL $\%$ according to the 5 -score harm scale. The confidence intervals (CIs) from scores 1 to 5 better describe positioning and dispersion of values. We can see some outliers in scores from 2 to 5 , and some overlapped values in distinct consecutive rows. To confirm that the harm scale makes a separation among different values of ECL $\%$, even so with a certain grade of overlapped values, we carried out the one-way ANOVA test $(\mathrm{P}<0.001)$ to demonstrate that the mean values of ECL\% for each score are different. The Tukey pairwise comparison test 

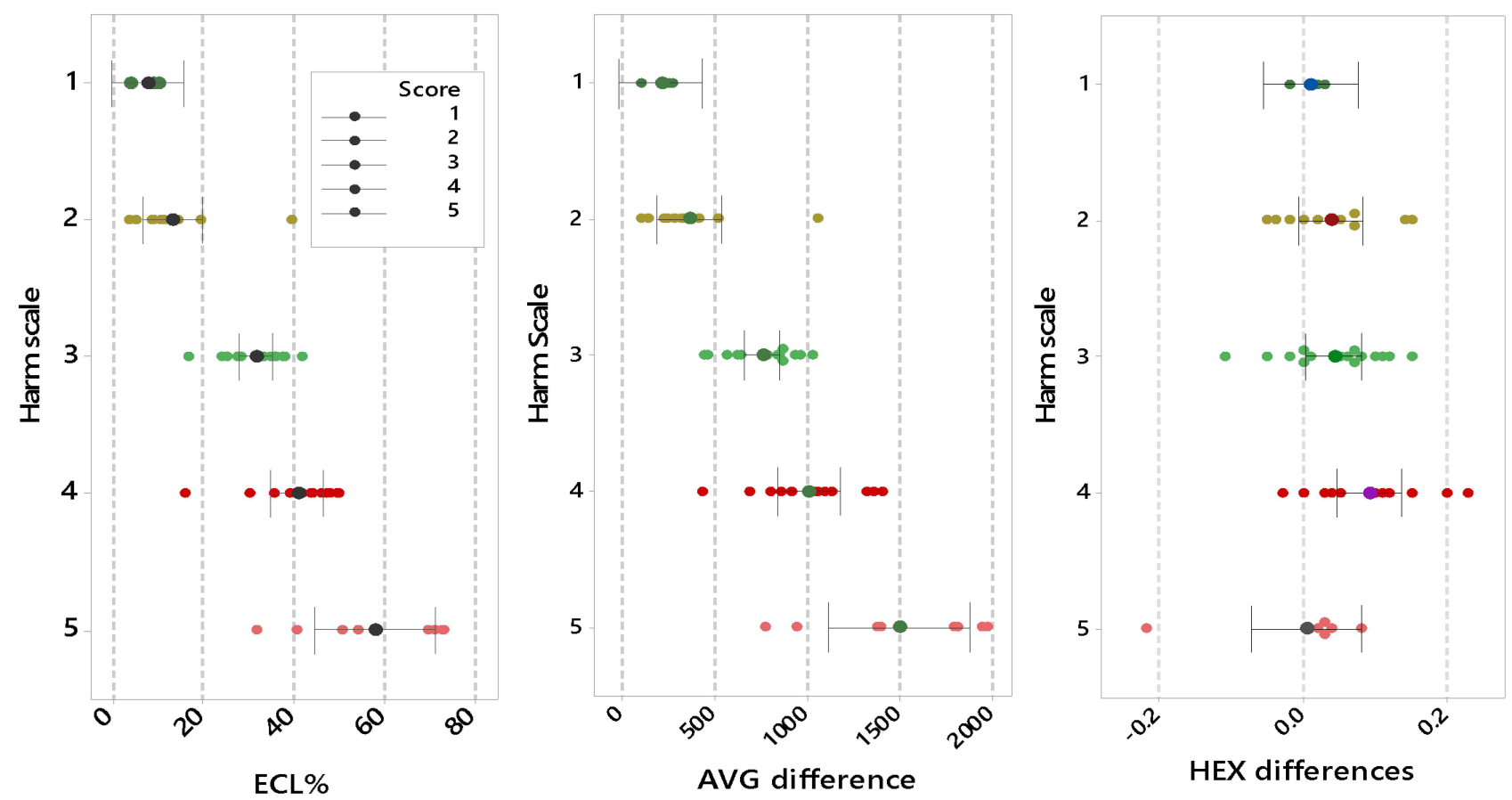

Figure I Interval plots. Single values and 95\% confidence interval plots of ECL\%, AVG\% and HEX\% depending on the 5-score harm scale. Abbreviations: ECL\%, percentage of endothelial cell loss; AVG\%, percentage of average cell area; HEX\%, percentage of hexagonality.

confirmed the overlapping of ECL\% for the 1-2 scores and 3-4, whereas score 5 remained distinct.

The second graph in Figure 1 (middle) describes the increasing trend of $\mathrm{AVG} \%$, once again depending on the 5 -score harm scale. Unlike ECL\%, this trend is more gradual for scores 1-4 and steeper for score 5 showing an exponential-like trend. Even here, there are some superimpositions among distinct scores and some outliers. The one-way ANOVA test on AVG\% $(\mathrm{P}<0.001)$ showed that the mean values were different. Tukey pairwise comparison test confirmed a partial overlapping of scores 1-2 and $3-4$, and a definitely separation of score 5 . The third graph in Figure 1 (right) shows that trend of HEX\% does not reflect the increasing trend of the 5-score harm scale. The different 95\% CIs are largely overlapped, while several values are totally scattered. We can see a slight tendency of decreasing $\mathrm{HEX} \%$ in scores from 1 to 4 , but in score 5 data points are irregularly scattered along the row. Anyway, we can see an irregular distribution of values almost for each score. The analysis of variance (one-way ANOVA at $\alpha=0.05$ ) showed that there is no difference among the mean HEX\% for scores $1-5(\mathrm{P}<0.092)$.

Considering the fact that the international scientific community usually takes into consideration the ECL\%, the most detectable and reliable indicator of the supposed harm on the endothelium, we first wanted to correlate this value with $\mathrm{AVG} \%$ and then with $\mathrm{HEX} \%$. In the first case (Figure 2), the relationship was given in a quadratic model $(\mathrm{P}<0.0001)$. When we have low ECL\% (score from 1 to 3 of the harm scale), this correlates to $\mathrm{AVG} \%$ average increase less or equal to $100 \%$; also, when we find high ECL\% (4 or 5 score of the harm scale), this means remarkable AVG\% increase up to more than $250 \%$.

The relationship between HEX\% and ECL\% (Figure 3) was characterized by a great variability and no specific

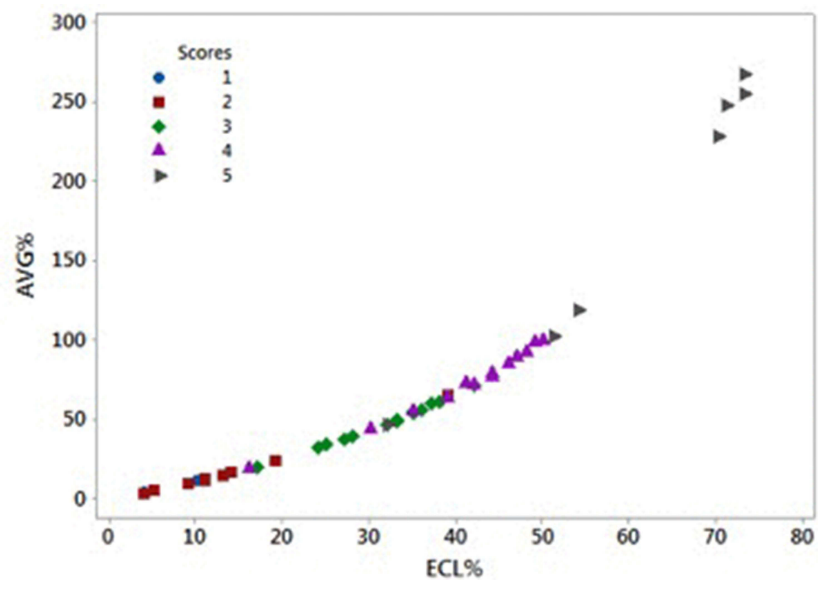

Figure 2 The quadratic trend of AVG\% depending on ECL\%. This trend can be described with two lines: a flat one in the first part up to ECL\% around $50 \%$ and AVG\% around $100 \%$, and a steep one for the second part of the curve.

Abbreviations: ECL\%, percentage of endothelial cell loss; AVG\%, percentage of average cell area. 


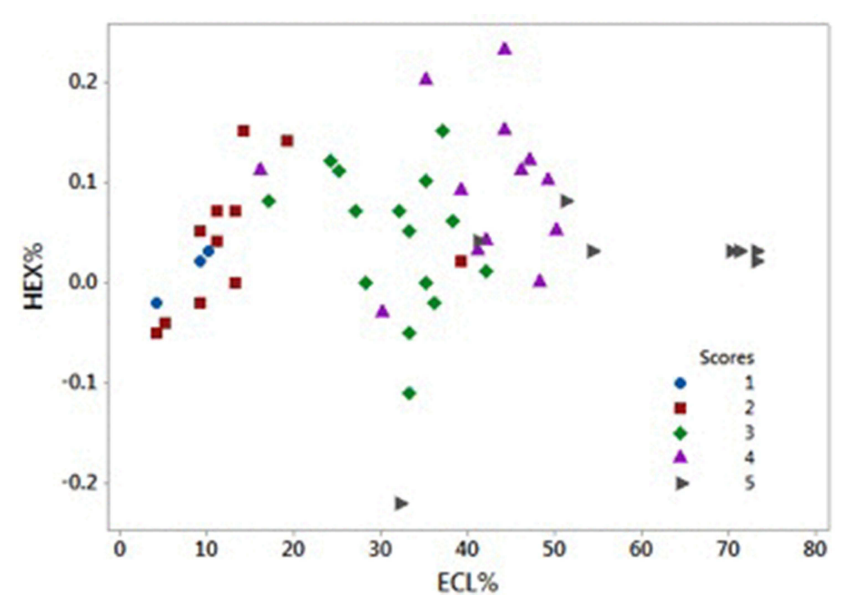

Figure 3 Scatter plot of HEX\% depending on ECL\%. There is no evidence of a specific relation between variables.

Abbreviations: ECL\%, percentage of endothelial cell loss; HEX\%, percentage of hexagonality.

trend could be recognized. In other words, low or high ECL\% could correspond to any loss of hexagonality, regardless of the score of the harm scale.

The 5-score harm scale is also a tool to evaluate energy delivered into the anterior chamber analyzing the alterations of the endothelium, as first stated in a clinical study in $2016 .^{6}$ In the above-mentioned study, authors have just observed quantitative changes in ECs, but this time we wanted to analyze qualitative changes in ECs. In Figure 4, we named the energy produced during sculpting as Phaco1 and the one used during quadrant removal as Phaco2. There was a positive correlation between the two energy-delivering phases of phaco. For each bubble, there are two main features: color and diameter. The first relates to the score of the harm scale, while the second to the size of the analyzed variable (ECL\%, $\mathrm{AVG} \%$ or $\mathrm{HEX} \%$ ). Thus, colors have been used to categorize the cataracts according to the 5-score harm scale, whereas the bubble width indicated changes in the number, average area or hexagonality of ECs. The narrower the diameter, the smaller $\mathrm{ECL} \%$ or $\mathrm{AVG} \%$ or $\mathrm{HEX} \%$; the larger the diameter, the bigger ECL $\%$ or $\mathrm{AVG} \%$ or $\mathrm{HEX} \%$. The distinct bubbles, connected with specific values of Phacol and Phaco2, asymmetrically spread out over the bisector of the scatterplot. Values with equal energy lie on the bisector. The bisector splits the plane: almost all bubbles significantly lie over the up left half-plane, where values of Phaco2 are higher than matching values of Phaco1. Phaco 1 and Phaco 2 are linearly related $(\mathrm{r}=1.5, \mathrm{P}<$ $\left.0.001, r^{2}=79 \%\right)$. As a consequence, the spatial distribution of the three indicators of endothelial alterations ECL\%, AVG\%, HEX\% - mostly depends on the energy delivered during Phaco2.

\section{Discussion}

As well known, during cataract surgery the phaco power modulation delivers energy into the anterior chamber. This energy mainly affects three measured parameters: ECL (surgically induced endothelial cell loss), AVG and HEX. If we consider the recent classification of the 5-score "harm scale" proposed by Sorrentino et al, each of these variables depends on both the grade of hardness of the cataract and the intraoperative phaco times. ${ }^{6}$
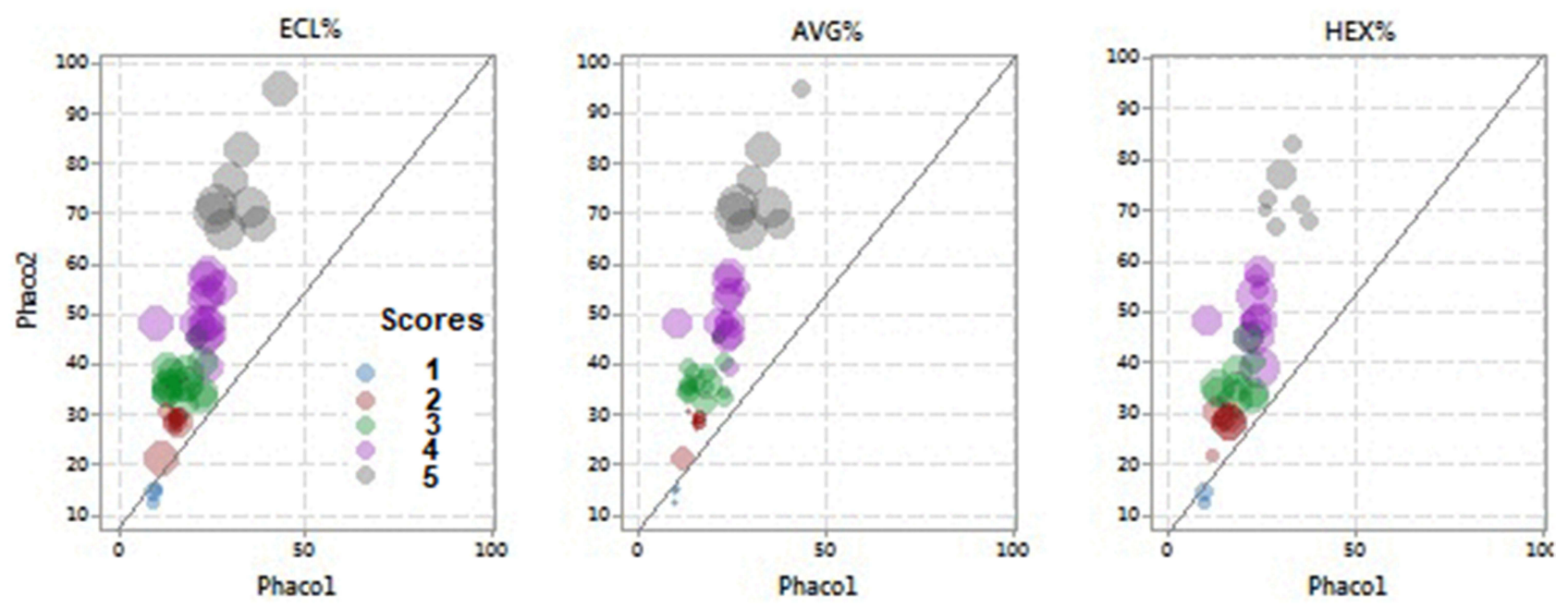

Figure 4 Bubble plots of Phacol and Phaco2. To analyze energies delivered into the anterior chamber during phacoemulsification according to ECL\%, AVG\%, HEX\%. Abbreviations: Phacol, phacoemulsification time for nucleus cracking; Phaco2, phacoemulsification time for quadrant removal; ECL\%, percentage of endothelial cell loss; AVG\%, percentage of average cell area; HEX\%, percentage of hexagonality. 
The "harm scale" is a new method that has been first suggested by Sorrentino et al in 2016, and secondly in 2017 , to categorize distinct cataracts according to preoperative and intraoperative variables: grade of hardness and duration of phaco times, respectively. ${ }^{6}$ This method would be useful to establish a connection between the type of cataract and the predictable harm on corneal endothelium after longitudinal phaco. Another pilot study applying the method of the harm scale to torsional phaco is in progress. The harm scale has been constructed giving a score from 1 to 5 to each cataract (Table 1).

In this study, our goal was first to describe the quantitative and qualitative changes of ECs after longitudinal phaco and secondly to analyze the way the energy delivered into the anterior chamber affects changes in both polymegathism and pleomorphism of ECs.

The non-contact specular microscopy gives three main indicators about corneal endothelium: ECD, AVG and HEX. Globally well recognized and internationally well accepted, the ECL\% is the main factor that has been taken into account in the harm scale. The AVG\%, closely linked to ECL\%, gives similar information with different trends and variables (Figure 2). Concerning the $\mathrm{HEX} \%$, there is no clear relationship depending on ECL\%, perhaps because it is the less detectable and measurable among the main indicators of the endothelium status (Figure 3). Therefore, we confirm that there might be a mechanism, somehow already hypothesized, according to which the loss of hexagonality for high scores is biologically counterbalanced by ECs retrieving the best hexagonal shape after phaco as much as possible.

The hardness grading is a preliminary approximate evaluation of any cataract. There is always a sort of uncertainty linked to the subjective assessment of the ophthalmologist who makes the preoperative examination. Conversely, phaco times are objective measurements intraoperatively taken. We chose the time instead of the energy because the phaco machine (Optikon R-Evolution) gave us the duration of each phase of phaco. And it is well known that the time corresponds to the energy (Energy = Power $\times$ Time).

Observing graphs in Figure 1, there were some outliers that could be attributed to the hard construction of the harm scale, supposedly due to the difficulty of the preoperative assessment of hardness grading. The algorithm measuring $\mathrm{AVG} \%$ is less sensitive than one analyzes ECL\%, because morphological characteristics are much more complex to detect than just a number of cells. Thus, the measurement of AVG\% might be less precise and the scale could be less reliable. Concerning HEX\%, we observed a light decreasing trend in hexagonality of ECs for scores 1-4. The HEX\% seen in score 5 is of difficult understanding. Anyway, the variations of HEX\% were so tiny that they might depend on the limit of the machine sensitivity. Geometric conditions of cells, such as the hexagonality, are very hard to detect by statistical algorithms.

Observing the bubble plots in Figure 4, we appreciated that the main damage on ECs was due to the second phase of phaco (Phaco2). In fact, most of bubbles for ECL\%, $\mathrm{AVG} \%$ and $\mathrm{HEX} \%$ spread across the upper half plane, where Phaco2 was higher than Phacol. In the bubble plots of AVG\% and HEX\%, bubbles were small and rather overlapped. This is likely to be attributed to the way of measuring cell morphology, less defined than the algorithm for measuring the quantity/density of cell loss.

Table I The 5-Score Harm Scale and the Quantitative/Qualitative Changes of Endothelial Cells for Increasing Scores

\begin{tabular}{|c|c|c|c|c|c|}
\hline Score of Harm Scale & I & 2 & 3 & 4 & 5 \\
\hline Hardness & I & $\mathrm{I}-2$ & $2-3$ & $3-4$ & 4 \\
\hline Phacol & $<10$ & $<16$ & $<22$ & $<28$ & $\geq 28$ \\
\hline Phaco2 & $<15$ & $<30$ & $<45$ & $<60$ & $\geq 60$ \\
\hline Number of patients & 3 & 11 & 15 & 13 & 8 \\
\hline $\mathrm{ECL} \%$ Mean $\pm \mathrm{SE}$ & $7.67 \pm 1.86$ & $13.36 \pm 2.85$ & $31.67 \pm 1.66$ & $40.85 \pm 2.61$ & $58.13 \pm 5.66$ \\
\hline AVG\% Mean \pm SE & $8.32 \pm 2.09$ & $16.97 \pm 5.05$ & $47.47 \pm 3.40$ & $72.46 \pm 6.37$ & $178.10 \pm 29.30$ \\
\hline HEX\% Mean \pm SE & $0.01 \pm 0.01$ & $0.04 \pm 0.02$ & $0.04 \pm 0.02$ & $0.09 \pm 0.02$ & $0.01 \pm 0.03$ \\
\hline
\end{tabular}

Abbreviations: Phacol, phacoemulsification time (s) to do nucleus cracking; Phaco2, phacoemulsification time (s) to do quadrant removal; ECL, endothelial cell loss; SE, standard error; AVG, average cell area; HEX, hexagonality. 
Probably, it might be argued that the sample size of our research was not very large. Certainly, bigger sample size is likely to give the chance to fine-tune the harm scale, avoiding the dispersion of some values in high scores.

In conclusion, the harm scale is a good strategy to foresee qualitative (average cell area and hexagonality) and quantitative (endothelial cell density) changes on corneal endothelium after longitudinal phaco. Anyway, new research is needed to prove the validity of the method of the harm scale with other phaco techniques or other phaco energy mode settings such as torsional. ${ }^{17,18}$

Finally, we confirmed that the most damage on ECs is due to the second phase of phaco, when most of the energy is delivered into the anterior chamber (less powerful ultrasounds but for longer time). However, further investigation is to carry out to try to establish connections among fluidics, vacuum, energy and final harm on corneal endothelium.

\section{Acknowledgments}

This research was conducted ethically in accordance with the World Medical Association Declaration of Helsinki. Subjects have given their written informed consent. This study protocol was approved by the institute's committee on human research.

\section{Author Contributions}

The author made a significant contribution to the work reported, whether that is in the conception, study design, execution, acquisition of data, analysis and interpretation, or in all these areas; took part in drafting, revising or critically reviewing the article; gave final approval of the version to be published; have agreed on the journal to which the article has been submitted; and agree to be accountable for all aspects of the work.

\section{Funding}

This research received no external funding.

\section{Disclosure}

The author reports no conflicts of interest.

\section{References}

1. Bourne RR, Minassian DC, Dart JK, Rosen P, Kaushal S, Wingate N. Effect of cataract surgery on the corneal endothelium: modern phacoemulsification compared with extracapsular cataract surgery. Ophthalmology. 2004;111:679-685.
2. Schmidt S, Hubich S, Vetter JM, Wirbelauer C. Effectivity and safety of a modified tip design in torsional phacoemulsification. Klin Monbl Augenheilkd. 2018;235(8):889-893. doi:10.1055/s-0043-124083

3. Packer M, Fishkind WJ, Fine IH, Hoffman RS. The physics of phaco: a review. J Cataract Refract Surg. 2005;31:424-431. doi:10.1016/j. jcrs.2004.11.027

4. Choi JY, Han YK. Long-term (10 years) results of corneal endothelial cell loss after cataract surgery. Can J Ophthalmol. 2019;54 (4):438-444. doi:10.1016/j.jcjo.2018.08.005

5. Al-Mohtaseb Z, He X, Yesilirmak N, Waren D, Donaldson KE. Comparison of corneal endothelial cell loss between two femtosecond laser platforms and standard phacoemulsification. $J$ Refract Surg. 2017;33(10):708-712. doi:10.3928/1081597X-20170731-01

6. Sorrentino FS, Bonifazzi C, Parmeggiani F, Perri P. A pilot study to propose a "harm scale", a new method to predict risk of harm to the corneal endothelium caused by longitudinal phacoemulsification, and the subsequent effect of endothelial damage on post operative visual acuity. PLoS One. 2016;11:e0146580.

7. Bourne WM. Biology of the corneal endothelium in health and disease. Eye. 2003;17(8):912-918. doi:10.1038/sj.eye.6700559

8. Yee RW, Matsuda M, Schultz RO, Edelhauser HF. Changes in the normal corneal endothelial cellular pattern as a function of age. Curr Eye Res. 1985;4(6):671-678. doi:10.3109/02713688509017661

9. Kageyama T, Hayashi R, Hara S, et al. Spontaneous acquisition of infinite proliferative capacity by a rabbit corneal endothelial cell line with maintenance of phenotypic and physiological characteristics. J Tissue Eng Regen Med. 2017;11(4):1057-1064. doi:10.1002/ term. 2005

10. Shimazaki J, Den S, Satake Y, Higa K. Continuous acellular material accumulation in the anterior chamber associated with corneal endothelial changes. BMJ Case Rep. 2020;13(11):e237417. doi:10.1136/bcr-2020-237417

11. Szalai E, Németh G, Berta A, Módis L. Evaluation of the corneal endothelium using noncontact and contact specular microscopy. Cornea. 2011;30:567-570. doi:10.1097/ICO.0b013e3182000807

12. McCarey BE, Edelhauser HF, Lynn MJ. Review of corneal endothelial specular microscopy for FDA clinical trials of refractive procedures, surgical devices, and new intraocular drugs and solutions. Cornea. 2008;27:1-16. doi:10.1097/ICO.0b013e31815892da

13. Chylack LT Jr, Leske MC, McCarthy D, Khu P. Lens Opacities Classification System II (LOCS II). Arch Ophthalmol. 1989;107:991-997. doi:10.1001/archopht.1989.01070020053028

14. Nair S, Nair RU. Wound and surface temperatures in vivo in torsional and longitudinal modalities of ultrasound in coaxial microincisional cataract surgery. Clin Ophthalmol. 2017;27(11):249-255. doi:10.2147/OPTH.S123222

15. Devgan U. Phaco fluidics and phaco ultrasound power modulations. Ophthalmol Clin North Am. 2006;19:457-468. doi:10.1016/j. ohc. 2006.08 .001

16. Doors M, Berendschot TT, Touwslager W, Webers CA, Nuijts RM. Phacopower modulation and the risk for postoperative corneal decompensation: a randomized clinical trial. JAMA Ophthalmol. 2013;131:1443-1450. doi:10.1001/jamaophthalmol.2013.5009

17. Vetter JM, Wirbelauer C. Improvement of phaco tip geometry during torsional phacoemulsification. Klin Monbl Augenheilkd. 2018;235 (5):611-615. doi:10.1055/s-0043-106298

18. Demircan S, Gokce G, Atas M, Baskan B, Goktas E, Zararsiz G. The impact of reused phaco tip on outcomes of phacoemulsification surgery. Curr Eye Res. 2016;41(5):636-642. doi:10.3109/ 02713683.2015.1039654 


\section{Publish your work in this journal}

Clinical Ophthalmology is an international, peer-reviewed journal covering all subspecialties within ophthalmology. Key topics include: Optometry; Visual science; Pharmacology and drug therapy in eye diseases; Basic Sciences; Primary and Secondary eye care; Patient Safety and Quality of Care Improvements. This journal is indexed on PubMed
Central and CAS, and is the official journal of The Society of Clinical Ophthalmology (SCO). The manuscript management system is completely online and includes a very quick and fair peer-review system, which is all easy to use. Visit http://www.dovepress.com/ testimonials.php to read real quotes from published authors.

Submit your manuscript here: https://www.dovepress.com/clinical-ophthalmology-journal 